\title{
On Collapse of Quantum State on Measurement
}

\author{
Basudev Nag Chowdhury ${ }^{1, *}$, Sanatan Chattopadhyay ${ }^{1}$ \\ ${ }^{1}$ Department of Electronic Science, University of Calcutta \\ *E-mail address: basudevn@gmail.com
}

\begin{abstract}
:
In the context of the century-long debate on quantum measurement problem, the current work proposes a model that describes the process of collapse of state by quantum interaction, which resolves the controversies of the framework of quantum mechanics and describes the entire domain of quantum-to-classical world including the weak measurement and partial collapse. 'Measurement', being the process of physically interacting with a system in order to extracting information from it, is theorized in the current model by synthesizing the quantum interaction between system and measuring apparatus with the information entropy of such process. The model assumes Schrödinger equation to be the only guiding equation for all physical systems including the measuring apparatus, and does not presuppose 'superposition principle', rather derives it theoretically from the formulation. The superposed state is shown to be independent of the choice of measurement operator (observable) or basis states (pointers) of the measuring apparatus. Most interestingly, the current model explains the non-observance of 'superposition principle' by classical systems as the classical limit of such quantum description of measurement. Along with solving the quantum measurement problem, the work also explains weak measurement and partial collapse, which can be further extended to investigate such several emerging critical phenomena.
\end{abstract}

Keywords: Quantum measurement problem; Collapse of state; Superposition principle; Information entropy. 
The concept of 'Measurement' for quantum objects has been a subject of century-long debate [1]. The central idea regarding such quantum measurement is that the quantum state of a system, while performing a single measurement of an observable on it, collapses into one of the eigenstates of the observable, which is a definite state of the measuring apparatus; and measured value of the observable is statistical average of the results of a large number of such measurement runs [2]. The quantum system is believed to be in a linear superposition of all such eigenstates of the observable, which, however, collapses on measurement irreversibly and randomly to any one of them. On the other hand, the measuring apparatus being a macroscopic object is supposed to be in a definite state and not a superposed one. Such model has been observed to be consistent and in full agreement with all the quantum phenomena in practice, however, led to a number of contradictory issues from theoretical perspective [3]. This raises, first and foremost, the question why superposition principle is not valid for macro-objects like the measuring apparatus which should belong to the classical limits of quantum conditions. This in turn poses the question, where is the boundary line between the quantum and classical world? Secondly, since it is a subjective choice to measure an observable of a system, how the system remains in a linear superposition of the eigenstates of that very observable in a predetermined way? Or does it simultaneously exist in the superposition of eigenstates of all the possible observables? Thirdly, the evolution of a quantum system is guided by Schrödinger equation in a so-called deterministic manner; however, probability comes into play when a measurement is performed and then the system is guided by Born rule. Quantum theory, as per Copenhagen interpretation [4], has not provided any specific dynamics for such transition.

Numerous attempts, such as decoherence, many-worlds interpretation, Bohmian model and the latest GRW and other spontaneous collapse models, have been made to resolve such seemingly contradictory issues $[\mathbf{3}, \mathbf{5 - 6}]$. Such theories are proposed on the basis of two requirements:

1) The system (supposed to be quantum) and the measuring apparatus (supposed to be classical) both are subsystems of the inseparable world and thus must be described by the same equation of motion including the measurement process; and

2) If Schrödinger equation is incapable to inclusively explain such measurement process, then either it does not provide a complete description of the nature and needs additional 
variables, or otherwise, a modified equation; and/or during measurement, the system jumps to one of the eigenstates being guided by some other equation(s) of motion which is(are) still to be known.

Thus, such efforts have been in search of either 'hidden variables' or entirely new law(s) of motion with quantum mechanics as its limiting/special case. However, no general consensus has been developed to reach any conclusion till date.

With the abovementioned issues, the authors want to add another seemingly contradictory point to the model of quantum mechanics. According to the Born rule if a system is in state $|\psi\rangle$, its 'probability amplitude' to be in a state $|\varphi\rangle$ is the inner product $\langle\varphi \mid \psi\rangle$, the corresponding 'probability' being $|\langle\varphi \mid \psi\rangle|^{2}$. However, for identical states the 'probability' is given by $\langle\psi \mid \psi\rangle$ and not by $|\langle\psi \mid \psi\rangle|^{2}$. Thus if it is considered the state $|\varphi\rangle$ to be $|\varphi\rangle=|\psi+\Delta \psi\rangle$, as one moves from $\Delta \psi \rightarrow 0$ to $\Delta \psi=0$, the 'probability amplitude' $\langle\varphi \mid \psi\rangle$ jumps to be 'probability'. Or otherwise, 'quantum probability' is restricted to be 'normalized' unlike the conventional statistical probability (which may be un-normalized in general), and indeed, is different from the latter one which needs further enquiry.

In this context, the current work proposes a model that redefines the measurement scheme within the framework of quantum mechanics that can lead to resolve the measurement problem in general. Such model does not assume 'superposition principle' as an ontological description of a quantum state, rather, explores the general process of quantum measurement which inevitably leads to the 'superposition principle'. The current model considers Schrödinger equation to be the guiding equation for all physical systems, be it the system subjected to measurement or be it the systems belonging to measuring apparatus.

Such proposed model assumes that if two isolated systems are exposed before each other to interact, there can be two kinds of possible inactions: one, damps one of the system's own prestate (i.e. the state when isolated); and the other does not. Only the former type of interaction, say Type-I, can be utilized for measurement; and thus call the other one Type-II. According to the current model, for measurement of a particular observable to be performed on a system, the 
relevant measuring apparatus needs to consist of a number of 'mutually non-interacting' (i.e. which, even if exposed before each other, do not interact) physical systems being in several different states, each of which can interact with the system in of Type-I manner to damp its prestate. When such measuring apparatus is entirely exposed before the system in a given state (i.e. pre-select), it selects one of the systems of measuring apparatus in a statistical probabilistic manner depending on their strength of interaction. It is imperative to mention that, for such 'mutually non-interacting' systems belonging to the measuring apparatus, the system subjected to measurement cannot but select only one of them to interact in type-I manner [for detail see Appendix at the end]. Once the selection is done, the state of system (i.e. post-select) on which measurement is being performed suffers a transition, or the so-called 'collapse', to that of the selected system of measuring apparatus. The present model considers that the probability amplitude of such post-selected transition can be described by the conventional 'inner product' in Hilbert space.

Suppose an isolated physical system ' $S$ ' in a definite state $|\psi\rangle$ with energy $\varepsilon_{S}$ is described by Schrödinger equation,

$i \hbar \frac{d}{d t}|\psi\rangle=\varepsilon_{S}|\psi\rangle$

and, an observable ' $O$ ' of the system is to be measured. Then formal quantum mechanics considers a particular choice of a set of non-degenerate orthonormal eigenstates $\left\{\left|\varphi_{j}\right\rangle, j=1,2, \ldots\right\}$ of the operator $\Theta$ (i.e. $\Theta\left|\varphi_{j}\right\rangle=\mathrm{O}_{j}\left|\varphi_{j}\right\rangle$ ) that corresponds to the measurement of observable ' $O$ '. According to the current model, in order to develop a corresponding measuring apparatus for measurement of ' $O$ ', firstly, one needs to prepare a set of 'mutually non-interacting' physical systems ' $I_{j}$ ' in definite states $\left|\zeta_{j}\right\rangle=\left|\varphi_{j}\right\rangle e^{-\left(\frac{i}{\hbar} \varepsilon_{j} t\right)}$ with energy values $\varepsilon_{j}$, respectively. Thus, all of such physical systems are also described by the same Schrödinger equation as,

$i \hbar \frac{d}{d t}\left|\zeta_{j}\right\rangle=\varepsilon_{j}\left|\zeta_{j}\right\rangle$ 
Thus, irrespective of the fact whether the operator $\Theta$ commutes with the Hamiltonian of system ' $S$ ' or not, it must commute with the Hamiltonian of the systems ' $I j$ '. Secondly, another necessity for the set of such physical systems to perform as measuring apparatus is that all of them must interact with the system ' $S$ ' only in Type-I manner, as mentioned earlier, in order to damp its pre-state $|\psi\rangle$. In fact, mathematically the operator $\Theta$ and its eigenvalues $\mathrm{O}_{j}$ are related to such particular type of physical interactions. When these conditions are fulfilled, each individual of the set of such specific physical systems may be assigned 'grades' by choosing appropriate standards of units corresponding to the values of observable ' $O$ ' (i.e. eigenvalues $\mathrm{O}_{j}$ of the operator $\Theta)$; and such systems ' $I_{j}$ ' can then be considered as the 'pointer's of measuring apparatus.

To perform measurement, one needs to probe the system ' $S$ ' with each of the pointers ' $I_{j}$ ' and record the results and average out to get the final measurement value. Physically such probing implies that each of the systems ' $I j$ ' be exposed (to interact) before the system ' $S$ ' a large number of times in its pre-select state, and after selection, each measurement run is considered to be complete once the post-select state of the system ' $S$ ' collapses to the corresponding state of the pointer. Such several runs of the measurement can be done, either by re-preparing the system ' $S$ ' in its pre-select state $|\psi\rangle$ after each measurement run is complete, or by preparing a number of identical replicas of the system ' $S$ ' in the pre-select state $|\psi\rangle$. One now only needs to record the probability of collapse of the post-selected state of the system into each of the pointer states $\left|\zeta_{j}\right\rangle$, which will be shown in this work in connection to the information entropy, to be equal to the relative weights of the corresponding 'grades' to obtain the 'expectation value'. Therefore, the current model of measurement synthesizes the physical interaction between a system and the measuring apparatus including statistical selection of pointer by the system and its non-statistical collapse to the selected pointer's state with the information entropy of the entire process.

With this consideration, in order to develop the model of such collapse of the post-select state $|\psi(t)\rangle$ of the system into one of the pointer states $\left.\left\{\zeta_{j}(t)\right\rangle, j=1,2, \ldots\right\}$, for instance into the $n^{\text {th }}$ pointer state $\left|\zeta_{n}(t)\right\rangle$, suppose the systems ' $S$ ' and ' $I_{n}$ ' start interacting at time $t=0$. Thus the equations (1) and (2) are modified for the post-select states respectively by, 


$$
\begin{aligned}
& i \hbar \frac{d}{d t}|\psi(t)\rangle=\varepsilon_{S}|\psi(t)\rangle+\tau_{n}\left|\zeta_{n}(t)\right\rangle \\
& i \hbar \frac{d}{d t}\left|\zeta_{n}(t)\right\rangle=\varepsilon_{n}\left|\zeta_{n}(t)\right\rangle+\tau_{n} *|\psi(t)\rangle
\end{aligned}
$$

where $\tau_{n}$, a complex function in general, represents the interaction between ' $S$ ' and ' $I n$ ', which was zero at the isolated or pre-select condition. Therefore, the probability that ' $I_{n}$ ' out of all ' $I_{j}$ 's is selected for interaction by the system ' $S$ ' is given by the statistical definition as,

$$
p_{n}=\frac{\left|\tau_{n}\right|^{2}}{\sum_{j}\left|\tau_{j}\right|^{2}}
$$

It is worthy to mention at this point that Eq. (3) and (4) together represent Schrödinger equation for the combined system ' $S+I_{n}$ ' (may be written as $i \hbar \frac{d}{d t}\left(\begin{array}{l}|\psi(t)\rangle \\ \left|\zeta_{n}(t)\right\rangle\end{array}\right)=\left(\begin{array}{cc}\varepsilon_{S} & \tau_{n} \\ \tau_{n} * & \varepsilon_{n}\end{array}\right)\left(\begin{array}{l}|\psi(t)\rangle \\ \left|\zeta_{n}(t)\right\rangle\end{array}\right)$ ) which is in corroboration with the assumption that Schrödinger equation is the only guiding equation of motion for both the system as well as the apparatus. Considering the Green's function $\left(G_{n}\right)$ for ' $I_{n}$ ', the solution of Eq. (4) is given by,

$\left|\zeta_{n}(t)\right\rangle=\left|\varphi_{n}\right\rangle e^{-\left(\frac{i}{\hbar} \varepsilon_{n} t\right)}+\int d t_{1} G_{n}\left(t, t_{1}\right) \tau_{n} *\left|\psi\left(t_{1}\right)\right\rangle$

where, $\left(i \hbar \frac{d}{d t}-\varepsilon_{n}\right) G_{n}\left(t, t_{1}\right)=\delta\left(t-t_{1}\right)$. Since a phase $\left[e^{-\frac{i}{\hbar} \varepsilon_{n}\left(t-t_{1}\right)}\right]$ does not change any result on multiplying with delta function $\left[\delta\left(t-t_{1}\right)\right]$, the Green's function is given by $G_{n}\left(t, t_{1}\right)=-\frac{i}{\hbar} \theta\left(t-t_{1}\right) e^{-\left(\frac{i}{\hbar} \varepsilon_{n}\left(t-t_{1}\right)\right)}$. Putting the solution $\left|\zeta_{n}(t)\right\rangle$ given in (6) using such Green's function into Eq. (3), it is obtained to be,

$i \hbar \frac{d}{d t}|\psi(t)\rangle=\varepsilon_{S}|\psi(t)\rangle+\int d t_{1} \tau G_{n}\left(t, t_{1}\right) \tau_{n} *\left|\psi\left(t_{1}\right)\right\rangle+\tau_{n}\left|\varphi_{n}\right\rangle e^{-\left(\frac{i}{\hbar} \varepsilon_{n} t\right)}$ 
At this point, the requirement for the nature of interaction between the system and pointer of the measuring apparatus, i.e. of Type-I as mentioned earlier, is described as follows:

It is apparent that before the interaction starts the state of isolated system ' $S$ ' evolves in time as unitary process. However, once it starts interacting in Type-I manner with the measuring apparatus, the time evolution operator $\left(T\left(t, t_{0}\right)\right)$ of the system given by $|\psi(t)\rangle=T\left(t, t_{0}\right)\left|\psi\left(t_{0}\right)\right\rangle$ must have the property,

$T^{+}\left(t, t_{0}\right) T\left(t, t_{0}\right) \neq 1$

since the probability of finding ' $S$ ' in its initial post-select state no longer remains the same due to the process of collapse of such state while interacting. Nevertheless, the system and the pointer together (' $S+I_{n}$ ') evolves unitarily, the time evolution operator being

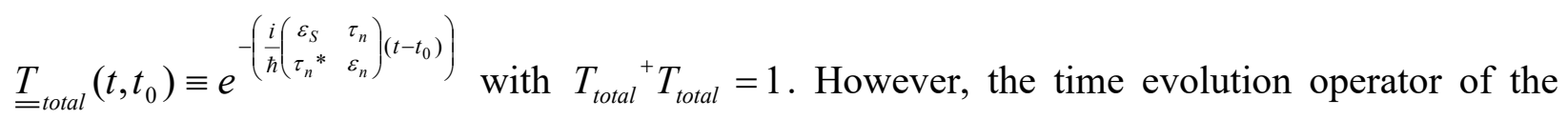
system ' $S$ ' only (a sub-part of the total system ' $S+I_{n}$ ') during such interaction is $T\left(t, t_{0}\right)=e^{-\left(\frac{i}{\hbar}\left(t-t_{0}\right) \Xi\right)}$ where $\Xi$ has a non-zero imaginary part (thus cannot be considered as the conventional Hamiltonian). Then considering the interaction to be independent of time, Eq. (7) leads to,

$$
\left.\left(i \hbar \frac{d}{d t}-\left[\varepsilon_{S}-\tau_{n}\left(\Xi-\varepsilon_{n}\right)^{-1} \tau_{n}^{*}\right]\right) \psi(t)\right\rangle=\tau_{n}\left|\varphi_{n}\right\rangle e^{-\left(\frac{i}{\hbar} \varepsilon_{n} t\right)}
$$

The solution of Eq. (9) is obtained to be

$$
|\psi(t)\rangle=|\psi(0)\rangle e^{-\frac{i}{\hbar}\left(\varepsilon_{S}-\tau_{n}\left(\Xi-\varepsilon_{n}\right)^{-1} \tau_{n}^{*}\right) t}+\left(\frac{\tau_{n}}{\varepsilon_{n}-\varepsilon_{S}+\tau_{n}\left(\Xi-\varepsilon_{n}\right)^{-1} \tau_{n} *}\right)\left|\varphi_{n}\right\rangle e^{-\left(\frac{i}{\hbar} \varepsilon_{n} t\right)}
$$

The condition for collapse is that the first term of such net solution, i.e. mathematically the complementary function and physically the pre-state of the system, damps out in time, which poses the restriction on imaginary part of $\tau_{n}\left(\Xi-\varepsilon_{n}\right)^{-1} \tau_{n} *$ to be positive. The second term, i.e. the particular integral, gives rise to the steady-state solution indicating the pointer state or the $n^{\text {th }}$ 
eigenstate of the operator $\Theta$. Thus, Eq. (9) represents a single measurement run which is equivalent to $\Theta|\psi\rangle=\mathrm{O}_{n}\left|\varphi_{n}\right\rangle$ and therefore $\Theta \equiv\left(i \hbar \frac{d}{d t}-\left[\varepsilon_{S}-\tau_{n}\left(\Xi-\varepsilon_{n}\right)^{-1} \tau_{n}{ }^{*}\right]\right)$ represents a general theoretical form of an operator in quantum measurement (' $\equiv$ ' is used since the dimension in general is not identical to that of the observable and the expression may need to be multiplied by a constant along with an ' $i=\sqrt{-1}$ ' in order to make it Hermitian). Different operators ' $\Theta$ ' are characterized by different kinds of interactions defined by ' $\tau$ '. The coefficient $\left(\frac{\tau_{n}}{\varepsilon_{n}-\varepsilon_{S}+\tau_{n}\left(\Xi-\varepsilon_{n}\right)^{-1} \tau_{n}^{*}}\right)$ of the steady state solution thus represents the probability amplitude of collapse of the post-select state of ' $S$ ' into that of ' $I n$ '. Hence, such probability amplitude must be equal to unity if $\varepsilon_{S}=\varepsilon_{n}$ irrespective of normalization, in order to resolve the apparent ambiguity raised by the authors earlier in this manuscript, which results to $\Xi=\varepsilon_{n}+\tau_{n}{ }^{*}$. Thus considering the real and imaginary parts of the interaction term as $\tau_{n}=\tau_{n}^{R}+i \tau_{n}^{I}$, the probability that the post-select state $|\psi(t)\rangle$ of the system while interacting with the pointer state $\left|\zeta_{n}(t)\right\rangle$ collapses to the latter is obtained to be,

$$
P_{n}=\frac{\tau_{n}^{R^{2}}+\tau_{n}^{I^{2}}}{\left(\varepsilon_{S}-\varepsilon_{n}-\tau_{n}^{R}\right)^{2}+\tau_{n}^{I^{2}}}
$$

which is maximum at $\varepsilon_{S}=\varepsilon_{n}+\tau_{n}^{R}$. A zero value of the real part $\tau_{n}^{R}$ of interaction term indicates the capability of measuring apparatus of 'accurately' measuring the observable and thus it can be considered as the instrumental error. Even if it is zero, if $\varepsilon_{S} \neq \varepsilon_{n}$, then there will be a lack of 'precision' due to the imaginary part of interaction which is a measure of the inherent uncertainty. It is to be noted that this is in complete agreement with the 'Copenhagen Interpretation' of quantum theory interpreting the inaccuracy in a measurement follows from the uncertainty relations as well as due to the difficulty of the experiment [4]. Therefore, to deal with the indeterminacy inherent in nature, such instrumental error is presently assumed to be zero, i.e. $\tau_{n}^{R}=0$ and $\Xi=\varepsilon_{n}-i \tau_{n}^{I}$, for further advancement of the model. The rate of collapse in such interaction can be obtained from the time evolution operator as $T^{+}(t, 0) T(t, 0)=e^{-\left(\frac{2 \tau_{\tau_{t}}^{t}}{\hbar}\right)}$. It can be 
seen that the essential difference of the current work from conventional 'Copenhagen Interpretation' of quantum measurement is that the process of collapse is not a discrete 'jump', but 'asymptotic' in time with the rate of collapse depending on the strength of interaction. For instance, if the interaction is of the order of ' $\mathrm{eV}$ ' in energy unit, the time constant for collapse $\left(\frac{\hbar}{2 \tau_{n}^{I}}\right)$ is of the order of $10^{-15} \mathrm{~s}$ and thus may be considered as mere 'jump' in practice. However, it can be much slower if the interaction is significantly weak and such case can interpret the partial collapse and /or the measurement of 'weak value' [7-10]. Very recently a few experiments have shown that the state of a system can evolve coherently and continuously during such so-called 'jump' transitions [11-12].

As mentioned earlier, the entire measurement process consists of two consecutive probabilities: first that the system ' $S$ ' in pre-select state selects a particular pointer ' $I_{n}$ ' statistically among all the ' $I_{j}$ 's for interaction given by (5), and second, if it selects to interact with ' $I_{n}$ ', then the probability that its post-select state $|\psi(t)\rangle$ collapses to $\left|\varphi_{n}\right\rangle$ in time as given by (11). Therefore, for the purpose of extracting information from such physical process involving conditional probability, the entropy of information can be written as [13],

$$
S=-\sum_{n} p_{n} \ln \left(P_{n}\right)
$$

Now, for the set of systems ' $I_{j}$ 's to be capable of working as the pointers of measuring apparatus, such entropy of information must be independent of any particular system ' $S$ ' in any particular pre-select state, and thus also of its energy $\varepsilon_{S}$, since the measuring apparatus must be proficient for performing measurement on any arbitrary system in any arbitrary pre-state, which requires $\frac{\partial S}{\partial \varepsilon_{S}}=0$. Thus, considering the instrumental broadening to be zero for ideal case, i.e. $\tau_{n}^{R}=0$, it results to, $\sum_{n}\left(\tau_{n}^{I^{2}} /\left[\left(\varepsilon_{S}-\varepsilon_{n}\right)^{2}+\tau_{n}^{I^{2}}\right]\right)\left(\varepsilon_{S}-\varepsilon_{n}\right)=0$, which can be rewritten as,

$$
\varepsilon_{S}=\frac{\sum_{n}\left(\tau_{n}^{I^{2}} /\left[\left(\varepsilon_{S}-\varepsilon_{n}\right)^{2}+\tau_{n}^{I^{2}}\right]\right) \varepsilon_{n}}{\sum_{n}\left(\tau_{n}^{I^{2}} /\left[\left(\varepsilon_{S}-\varepsilon_{n}\right)^{2}+\tau_{n}^{I^{2}}\right]\right)}
$$


It is now apparent from Eq. (13) that the energy of the system $\left(\varepsilon_{S}\right)$ is statistical 'expectation value' of energies of all the pointers $\left(\varepsilon_{n}\right)$ of the measuring apparatus, i.e. $\varepsilon_{S}=\left\langle\varepsilon_{n}\right\rangle$, if the probabilities of post-select state of the system to collapse into different pointer states (as given by (11) with $\tau_{n}^{R}=0$ ) are regarded as the relative weights of the corresponding 'grades'. This corroborates quite well with the conventional ontological description of 'superposition principle' of quantum mechanics and the principal essence of 'Copenhagen Interpretation'. Therefore, the current model of measurement scheme and process of collapse provides the information about the system, that, the pre-state $\left(|\psi\rangle\right.$ ) of system ' $S$ ' (with energy $\varepsilon_{S}$ ) can be expressed as a 'linear superposition' of the orthonormal states $\left(\left|\zeta_{j}\right\rangle\right)$ of a measuring apparatus pointers ' $I_{j}$ 's, the 'coefficients' of superposition being identical to the non-statistical probability amplitudes of collapses of the post-select states into the corresponding pointer states, if would be subjected to such measurement, and is given by,

$|\psi\rangle=\sum_{j}\left(\frac{i \tau_{j}^{I}}{\varepsilon_{j}-\varepsilon_{S}+i \tau_{j}^{I}}\right)\left|\zeta_{j}\right\rangle$

and the measured value will be the statistical expectation value of the observable. Interestingly, RHS of the expression given in (13) is identical to $\varepsilon_{S}$ independent of the choice of basis set of eigenstates of any arbitrary observable and thus the same is also valid for the expression of superposition given in (14). It also upholds that there is no restriction(s) on the pointer states of measuring apparatus to be expressed as a linear superposition of the eigenstates of any such observable. Further, beyond conventional formalism of quantum mechanics, (14) represents the analytical expression of coefficients of superposing states as a function of the system energy, pointer energy states of the measuring apparatus and their interactions, which obeys the 'normalization restriction' (i.e. equals 1 when $|\psi\rangle=\left|\zeta_{j}\right\rangle$ ) of the non-statistical probability. It is apparent that such result endorses that the quantum probability which is norm of such coefficients originates from physical interaction of quantum type and thus distinguishably different from the conventional statistical probability. Finally, it resolves the seemingly contradictory issue that a classical system does not exhibit superposition principle as follows: A classical system can be distinguished from a quantum object by the fact that the disturbance 
created by a measurement process on the former one is negligible. Thus at classical limit, the energy of interaction $\left(\tau_{j}^{I}\right)$ between the system and pointers of measuring apparatus is negligibly small compared to the order of their energy values $\left(\varepsilon_{S}, \varepsilon_{j}\right)$; and correspondingly the superposition principle of (14) results to,

$\left.\left.|\psi\rangle=\sum_{j} \underset{\tau_{j}^{L} \rightarrow 0}{L t}\left(\frac{i \tau_{j}^{I}}{\varepsilon_{j}-\varepsilon_{S}+i \tau_{j}^{I}}\right)\right) \zeta_{j}\right\rangle=\left\{\begin{array}{c}\left|\zeta_{j}\right\rangle \text { for } \varepsilon_{S}=\varepsilon_{j} \\ 0 \text { for } \varepsilon_{S} \neq \varepsilon_{j}\end{array}\right.$

which corroborates to the non-observance of superposition of states in classical reality.

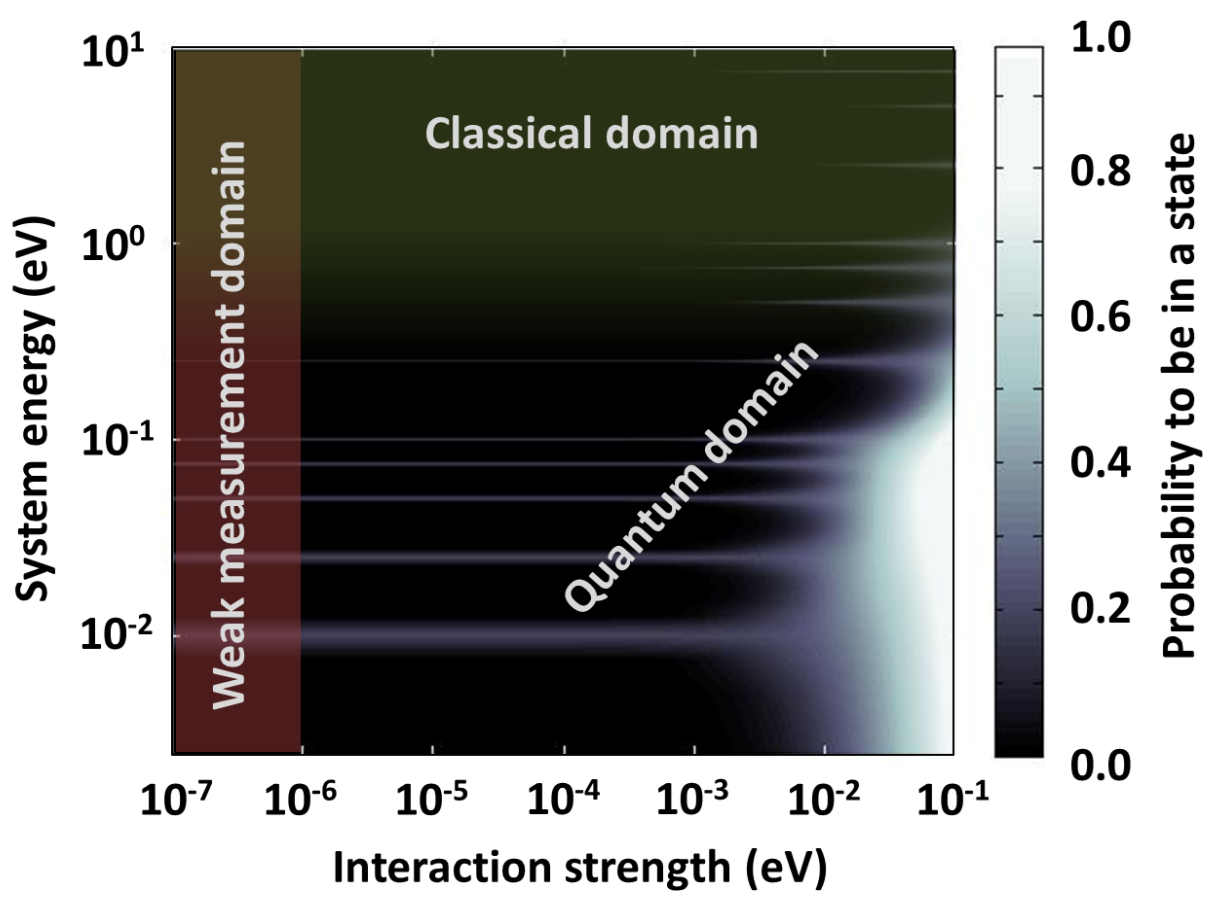

Fig.1. The plot of probability $(\langle\psi \mid \psi\rangle)$ of the system under measurement with and its energy $\left(\varepsilon_{S}\right)$ and the strength of interaction with the pointers $\left(\tau^{I}\right)$. The pointer states are considered to be of energies $\varepsilon_{n}=[0.10,0.25,0.50,0.75]$ of each order (powers of $10 \mathrm{in} \mathrm{eV}$ ) of energies.

The overall description of the measurement phenomenon as described in the current model is illustrated in Fig. 1 that encompasses entire domain of quantum to classical world including the weak measurement region. The figure represents how the probability of a system $(\langle\psi \mid \psi\rangle)$ varies 
with the order its energy $\left(\varepsilon_{S}\right)$ along with the strength of interaction $\left(\tau^{I}\right)$ with the pointers. It is apparent from the plot of Fig. 1, that for lower energy range (i.e. comparable to interaction strength), the probability is distributed into the pointer states with broadened intensity around them indicating superposition of such states thereby defining the quantum domain. However, with increasing energy (w.r.t. interaction strength), it is observed to be highly sharpened discretely at the pointer states only implying no-superposition as expected in classical reality. On the other hand, the region of interaction strength below the order of $\mu \mathrm{eV}$ of Fig. 1 represents the weak measurement and partial collapse phenomena considering collapse time of the order of z'ns'.

In conclusion, it can be said that, 'measurement', as an aim, implies some information to be extracted from a system, whereas 'measurement', as a process, involves a physical interaction with such system. Therefore, theorization of 'measurement' requires to synthesizing these two features, i.e. information and interaction, which is presented in the current work for interpretation of quantum measurement scheme which is in utter consonance with the classical limit. The work may provide a guide to find out answers to many questions regarding the century-long debate on the formalism of quantum mechanics including the so-called 'measurement problem' and can be further extended to study weak measurement, partial collapse and other such emerging critical phenomena.

\section{Appendix}

In order to show that the system subjected to measurement cannot select more than one pointers to interact simultaneously, let us assume hypothetically that the system interacts in type-I manner with more than one pointers. In such case, Eq. (3) would be given by,

$i \hbar \frac{d}{d t}|\psi(t)\rangle=\varepsilon_{S}|\psi(t)\rangle+\sum_{j} \tau_{j}\left|\zeta_{j}(t)\right\rangle$

which, instead of Eq. (9), would lead to

$\left.\left(i \hbar \frac{d}{d t}-\left[\varepsilon_{S}-\sum_{j} \tau_{j}\right]\right) \psi(t)\right\rangle=\sum_{n} \tau_{n}\left|\varphi_{n}\right\rangle e^{-\left(\frac{i}{\hbar} \varepsilon_{n} t\right)}$ 
It is imperative to note that $\tau$ 's in the RHS of Eq. (A-2) represent the inflow of interaction flux to the system from the corresponding pointers, whereas $\tau$ 's in LHS of such equation contributes to the corresponding outflow. Thus, the steady state of the system would be given by,

$|\psi(t)\rangle_{S t}=\int d t_{1} G_{S}\left(t, t_{1}\right)\left|S_{P}\left(t_{1}\right)\right\rangle$

where $\left|S_{P}(t)\right\rangle=\sum_{n} \tau_{n}\left|\varphi_{n}\right\rangle e^{-\left(\frac{i}{\hbar} \varepsilon_{n} t\right)}$ and $G_{S}\left(t, t_{1}\right)=-\frac{i}{\hbar} \theta\left(t-t_{1}\right) e^{-\left(\frac{i}{\hbar}\left[\varepsilon_{S}-\sum_{j} \tau_{j}\right]\left(t-t_{1}\right)\right)}$. Hence, considering $\sum_{P}\left(t_{1}, t_{2}\right)=\left\langle S_{P}\left(t_{2}\right) \mid S_{P}\left(t_{1}\right)\right\rangle$, it is obtained to be,

$\left\langle\psi\left(t^{\prime}\right) \mid \psi(t)\right\rangle_{S t}=\int d t_{1} \int d t_{2} G_{S}\left(t, t_{1}\right) \sum_{P}\left(t_{1}, t_{2}\right) G_{S}^{*}\left(t_{2}, t^{\prime}\right)$

Thus, the two-time flux $\Phi\left(t, t^{\prime}\right)=\left(\frac{d}{d t}-\frac{d}{d t^{\prime}}\right)\left\langle\psi\left(t^{\prime}\right) \mid \psi(t)\right\rangle_{S t}$ results to be

$$
\begin{array}{r}
\Phi\left(t, t^{\prime}\right)=\frac{1}{\hbar} \int d t_{1} \int d t_{2}\left[\sum_{j} i\left(\tau_{j}-\tau_{j}^{*}\right) G_{S}\left(t, t_{1}\right) \sum_{P}\left(t_{1}, t_{2}\right) G_{S} *\left(t_{2}, t^{\prime}\right)\right] \\
-\frac{i}{\hbar} \int d \bar{t}\left[\sum_{P}(t, \bar{t}) G_{S} *\left(\bar{t}, t^{\prime}\right)-G_{S}(t, \bar{t}) \sum_{P}\left(\bar{t}, t^{\prime}\right)\right]
\end{array}
$$

It is apparent that the first term in RHS of Eq. (A-5) represents the total outward flux $\left(\Phi_{\text {Out }}\left(t, t^{\prime}\right)\right)$ from system to all the pointers (note the term $\sum_{j} i\left(\tau_{j}-\tau_{j}{ }^{*}\right)$ ) while its second term indicates the total inward flux $\left(\Phi_{I n}\left(t, t^{\prime}\right)\right)$ accordingly from all the pointers to the system (note the term $\left.\sum_{P}\right)$. Neglecting the instrumental error, considering $\tau_{n}=i \tau_{n}^{I}$ and putting the expressions of $G_{S}$ and $\sum_{P}$, such inward and outward fluxes corresponding to a particular pointer, suppose the $n^{\text {th }}$ one, are respectively obtained to be,

$\left.\Phi_{I n}^{n}\left(t, t^{\prime}\right)=\frac{2 \sum_{k} \tau_{k}^{I}}{\hbar}\left[\frac{\left(\tau_{n}^{I}\right)^{2}}{\left(\varepsilon_{n}-\varepsilon_{S}\right)^{2}+\left(\sum_{j} \tau_{j}^{I}\right)^{2}}\right] e^{-\left(\frac{i}{\hbar} \varepsilon_{n}\left(t-t^{\prime}\right)\right.}\right)$

and 
$\left.\Phi_{\text {Out }}^{n}\left(t, t^{\prime}\right)=-\frac{2 \tau_{n}^{I}}{\hbar} \sum_{m}\left[\frac{\left(\tau_{m}^{I}\right)^{2}}{\left(\varepsilon_{m}-\varepsilon_{S}\right)^{2}+\left(\sum_{j} \tau_{j}^{I}\right)^{2}}\right] e^{-\left(\frac{i}{\hbar} \varepsilon_{m}\left(t-t^{\prime}\right)\right.}\right)$

It is observed from (A-6) and (A-7) that, if the system interacts with more than one pointers simultaneously (as assumed hypothetically), the net interaction flux between the system and any one of the pointers at steady state is not zero, i.e. $\Phi_{I n}^{n}\left(t, t^{\prime}\right)+\Phi_{\text {Out }}^{n}\left(t, t^{\prime}\right) \neq 0$. However, sum of such net interaction fluxes for all the interacting pointers is zero, i.e. $\sum_{n} \Phi_{I n}^{n}\left(t, t^{\prime}\right)+\Phi_{\text {Out }}^{n}\left(t, t^{\prime}\right)=0$. It implies that at steady state, net inflow of interaction fluxes from some of the interacting pointers to the system is equal to the net outflow of interaction fluxes to the rest of the interacting pointers. Such case is equivalent to 'mutual interaction' between the pointers with the system to be pseudo-present between them, which is in apparent contradiction to the basic assumption of 'mutually non-interacting' property of the pointers. However, if the system subjected to measurement selects only one pointer, the sums on all $\tau$ 's in (A-6) and (A7) are removed, which leads to the net steady state interaction flux to be zero, i.e. $\Phi_{I n}^{n}\left(t, t^{\prime}\right)+\Phi_{\text {Out }}^{n}\left(t, t^{\prime}\right)=0$.

\section{Acknowledgement}

Dr. Basudev Nag Chowdhury likes to acknowledge Center of Excellence (COE), TEQIP PhaseIII, World Bank for financial support and the Center for Research in Nanoscience and Nanotechnology (CRNN) for providing relevant infrastructural support to conduct the research work. 


\section{Reference}

1. Quantum Theory and Measurement, edited by J. A. Wheeler and W. H. Zurek, Princeton University Press, Princeton (1983).

2. P. A. M. Dirac, The Principles of Quantum Mechanics, Oxford University Press, London (1958), Chapter-I and II.

3. A. Bassi, K. Lochan, S. Satin, T. P. Singh, H. Ulbricht, "Models of wave-function collapse, underlying theories, and experimental tests", Rev. Mod. Phys., 85, 471 (2013).

4. W. Heisenberg, Physics and Philosophy, The Revolution in Modern Science, George Allen \& Unwin Ltd, London (1959).

5. M. Schlosshauer, "Decoherence, the measurement problem, and interpretations of quantum mechanics", Rev. Mod. Phys., 76, 1267 (2004).

6. J. S. Bell, Speakable and Unspeakable in Quantum Mechanics, Cambridge University Press, Cambridge (1987).

7. Y. Aharonov, D. Z. Albert, L. Vaidman, "How the Result of a Measurement of a Component of the Spin of a Spin- 2 Particle Can Turn Out to be 100", Phys. Rev. Lett., $60,1351(1988)$.

8. N. W. M. Ritchie, J. G. Story, R. G. Hulet, "Realization of a Measurement of a Weak Value", Phys. Rev. Lett., 66, 1107 (1991).

9. N. Katz et al, "Coherent State Evolution in a Superconducting Qubit from PartialCollapse Measurement”, Science, 312, 1498 (2006).

10. F. Piacentini et al, "Investigating the Efects of the Interaction Intensity in a Weak Measurement", Scientific Reports, 8, 6959 (2018).

11. Z. K. Minev et al, “To Catch and Reverse a Quantum Jump Mid-flight”, Nature, 570, 200 (2019). 
12. F. Pokorny et al, "Tracking the Dynamics of an Ideal Quantum Measurement", Phys. Rev. Lett., 124, 080401 (2020).

13. C. E. Shannon, "A Mathematical Theory of Communication", The Bell System Technical Journal, 27, 379 (1948). 